https://helda.helsinki.fi

\title{
Radial Diffusion of Planetary Radiation Belts' Particles by \\ Fluctuations with Finite Correlation Time
}

\section{Osmane, Adnane}

2021-05

Osmane , A \& Lejosne , S 2021, ' Radial Diffusion of Planetary Radiation Belts' Particles by

Fluctuations with Finite Correlation Time ' , Astrophysical Journal , vol. 912 , no. 2 , 142 . https://doi.org/10.3847/153

http://hdl.handle.net/10138/339567

https://doi.org/10.3847/1538-4357/abf04b

unspecified

publishedVersion

Downloaded from Helda, University of Helsinki institutional repository.

This is an electronic reprint of the original article.

This reprint may differ from the original in pagination and typographic detail.

Please cite the original version. 


\title{
Radial Diffusion of Planetary Radiation Belts' Particles by Fluctuations with Finite Correlation Time
}

\author{
Adnane Osmane $^{1}$ (1) and Solène Lejosne ${ }^{2}$ (1) \\ ${ }^{1}$ Department of Physics, University of Helsinki, Helsinki, Finland; adnane.osmane@ @elsinki.fi \\ ${ }^{2}$ Space Sciences Laboratory, Berkeley, CA, USA \\ Received 2020 December 7; revised 2021 March 15; accepted 2021 March 17; published 2021 May 14
}

\begin{abstract}
Radial diffusion in planetary radiation belts is a dominant transport mechanism resulting in the energization and losses of charged particles by large-scale electromagnetic fluctuations. In this study, we revisit the radial diffusion formalism by relaxing the assumption of zero correlation time in the spectrum of fluctuations responsible for the transport of charged particles. We derive a diffusion coefficient by assuming fluctuations that (1) are time homogeneous, (2) too small to trap the particles, and (3) can decorrelate on timescales comparable to the transit time of the particles. We demonstrate through self-similar solutions of the Fokker-Planck equation that autocorrelation time $\tau_{c}$ much larger than the linear transit time/particle drift period $\tau_{L}=\Omega_{D}^{-1}$ results in characteristic time for transport independent of the drift frequency and faster than for short correlation time. In both instances, that is for short $\left(\tau_{L} \gg \tau_{c}\right)$ and long $\left(\tau_{L} \ll \tau_{c}\right)$ autocorrelation time, the diffusion of particles is subdiffusive since the variance of increments along the magnetic drift shells $L^{*}$, scales as $\left\langle\Delta L^{* 2}\right\rangle \sim t^{s}$, with $s<1$. However, in the absence of sources and sinks, particle transport for both short and long autocorrelation times result in equilibrium distribution along $L^{*}$ with differences of less than $10 \%$ across lower magnetic drift shells. The main consequence of incorporating finite correlation time appears in intermediate times much longer than the drift period but before the distribution function reaches equilibrium and indicates the importance of quantifying observationally the spectral properties of fluctuations for the modeling of planetary radiation belts.
\end{abstract}

Unified Astronomy Thesaurus concepts: Plasma astrophysics (1261); Space plasmas (1544); Plasma physics (2089); Alfven waves (23); Van Allen radiation belt (1758)

\section{Introduction}

The interaction of the solar wind plasma with planetary magnetic fields gives rise to a cavity in which charged particles are magnetically confined and accelerated to relativistic energies. The confinement of these particles is the result of the geomagnetic field topology characterized by magnetic field lines that converge at high latitudes toward the poles. In the absence of electromagnetic perturbations, the motion of the trapped particles can be decomposed in terms of three adiabatic invariants. The first and second adiabatic invariants $\mu$ and $J$ map the quasi-periodic motion of the particle around and along the field line. The third adiabatic invariant, here denoted as $\Phi$, is the magnetic field enclosed by the azimuthal drift orbit. However, the quasi-periodic orbits can become chaotic in the presence of electromagnetic fluctuations that can violate one or more of the adiabatic invariants. Breaking one or multiple adiabatic invariants by electromagnetic fluctuations results in the irreversible transfer of energy from the fields to the particles. Observationally, this translates into the enhancement of suprathermal particles confined in planetary environments and known as radiation belts (Walt 2005).

In the Earth's radiation belts trapped electrons with energies of less than $1 \mathrm{keV}$ to $10 \mathrm{MeV}$ are commonly measured. Consequently, the Earth's radiation belts are the closest natural astrophysical laboratory in which we can track in situ the energization of charged particles to relativistic energies. The past decades of in situ measurements have highlighted two dominant mechanisms: radial diffusion by large-scale fluctuations that violate the third adiabatic invariant $\Phi$ (Lejosne \& Kollmann 2020) and local wave-particle interactions resulting in the violation of the first and second adiabatic invariants by kinetic-scale fluctuations (Ukhorskiy \& Sitnov 2013; Reeves et al. 2013; Thorne 2013). Incidentally, both mechanisms are stochastic and rely on equivalent assumptions to derive a Fokker-Planck equation in their respective phase space, that is, (1) time and space homogeneity of the force field fluctuations, (2) unperturbed particle orbits, and (3) correlation times much smaller than the transit time (Taylor 1922; Parker 1960; Sturrock 1966; Fälthammar 1965; Diamond et al. 2010; Adkins 2018). For radial diffusion the transit time corresponds to the azimuthal drift period, whereas for local wave-particle interactions in a magnetized plasmas it is the time for a particle with speed $v_{\|}$to cross one wavelength $\lambda_{\|}$, i.e., $\tau_{L} \simeq \lambda_{\|} / v_{\|}$. It is therefore common practice to consider that the characteristic time for the variations of the signal is very small in comparison with the transit time of the particles considered and to describe the effect of these field variations on a timescale that is greater than both the transit time of the particle, and the autocorrelation of the fluctuations, i.e., $\tau_{c} \ll \tau_{L} \ll t$.

However, unlike in fluid dynamics and for the specific problem treated by Taylor (1922), MHD fluctuations cannot simply random walk as this would have the consequence of increasing field-line tensions. In a stable plasma the field-line tension tries to return the fluid element to the unperturbed equilibrium position, and plasma fluid elements are therefore subject to a spring-back effect. This elasticity inherent to MHD plasmas has the consequence of allowing long-time correlations in the fluctuations. The consequences of relaxing the assumptions made by Taylor (1922) for particles experiencing radial diffusion (Fälthammar 1965) remains an open theoretical question relevant to modeling the Earth's radiation belts. The 
primary aim of this communication is to characterize the stochastic transport when the autocorrelation time becomes comparable $\left(\tau_{c} \simeq \tau_{L}\right)$ or much longer than the transit time $\left(\tau_{c} \gg \tau_{L}\right)$. Decay of the fluctuations as experienced by a particle interacting with a wave packet can be treated analytically by accounting for at least one of two separate processes. One such process originates in fluctuation amplitudes or wavevectors being stochastic while assuming that the particle orbits remain unperturbed. Another one is in the nonlinear scattering of the particle orbits while assuming that the fields' amplitudes remain constant. ${ }^{3}$

In particular, we would like to know if the equilibrium distribution and diffusion coefficient is affected by a nonzero correlation time of the fluctuations. On the basis of observational in situ measurements and numerical experiments the diffusion coefficient for radial transport spans four orders of magnitude and is strongly dependent on the geomagnetic activity (Brautigam \& Albert 2000; Huang et al. 2010). In a more recent study using Bayesian inference and the Van Allen Probe measurements, Sarma et al. (2020) empirically determined the parametric dependence of the diffusion coefficient. They find a bimodal distribution for the exponent of $L^{*}$ in the diffusion coefficient, with one peak centered above a value of 10 and another, slightly more pronounced, below 10 . To the best of our knowledge, a physical explanation for the parametric change of the diffusion coefficient reported by Sarma et al. (2020) in terms of the statistical properties of the electromagnetic fluctuations has not been studied. ${ }^{4}$ Our hypothesis is that the appearance of finite correlation times in the electromagnetic fluctuations could translate into a diffusion coefficient with a parametric dependence in the $L^{*}$ exponent consistent with in situ measurements.

In the following study we use the recipe of Taylor (1922) to quantify the transport of passive particles in a turbulent field. We modify the calculation of Taylor (1922) by only relaxing the assumption of zero autocorrelation times. ${ }^{5}$ In other words, we still assume that electrons sample small amplitude and stationary turbulent fluctuations as postulated by Taylor (1922). We use a simple model for the electromagnetic fields in order to be able to solve the transport equation analytically. In our particular case, the electromagnetic turbulence consists of Alfvénic fluctuations carried by the protons while the passive tracers are the electrons. The magnetic field corresponds to a background dipole field, to which small time-varying perturbations with a dependence on magnetic local time, here expressed in terms of phase $\varphi(t)$, are superimposed (Mead 1964; Fälthammar 1965). With a representation of the perturbation in terms of a symmetric component $S(t)$ and an antisymmetric one $A(t) r \cos (\varphi)$, it is possible to show that an ensemble of particles with adiabatic invariant $\Phi$ (Ukhorskiy \& Sitnov 2013), corresponding to the magnetic flux bounded by the drift contour of a trapped particle, can only experience irreversible

\footnotetext{
3 We resort to the first effect but the correlation time $\tau_{c}$ appearing in the diffusion coefficient (14) can encode decay occurring due to turbulent scattering as well. For a recent pedagogical discussion on the topic the reader can consult Bian et al. (2014) and find references therein.

4 A similar problem has been addressed for a Vlasov-Poisson system by Adkins (2018) that shows that it is possible to modify the properties of the diffusion coefficient by accounting for the finite correlation time of turbulent stochastic fluctuations.

5 The statement zero correlation time should not be taken literally but as a limit with respect to another timescale. In our case, the correlation time is zero in the limit where $t / \tau_{L} \gg \tau_{c} / \tau_{L} \rightarrow 0$, i.e., when the transit time is much longer than the autocorrelation time $\tau_{c}$
}

changes in the presence of nonzero antisymmetric fluctuations, i.e., $A(t) \neq 0$. $^{6}$ Symmetric fluctuations $S(t)$ do not contribute statistically to the change in the third adiabatic invariant (Lejosne 2019; Lejosne \& Kollmann 2020).

\section{Theoretical Model}

\subsection{Equation of Motion and Dispersion in $\mathrm{L}^{*}$}

The third adiabatic invariant $\Phi$ is the magnetic flux sampled by an azimuthally drifting orbit:

$$
\Phi=\int_{\mathcal{S}} \boldsymbol{B} \cdot \boldsymbol{n} d A=\int_{\Gamma} \boldsymbol{A} \cdot d \boldsymbol{l},
$$

in which the drift contour $\Gamma$ bounds the surface $\mathcal{S}$ upon which the flux of the magnetic field $\boldsymbol{B}=\nabla \times \boldsymbol{A}$ with magnetic potential vector $\boldsymbol{A}$ is computed. It is convenient to track the evolution of the third adiabatic invariant $\Phi$ in terms of $L^{*}$ since for a dipole magnetic field the latter corresponds to drift shell radial distance from the Earth's magnetic equatorial plane:

$$
L^{*}=\frac{2 \pi B_{E} R_{E}^{2}}{\Phi} .
$$

In the above equations, $B_{E}$ represents the amplitude of the equatorial magnetic field at one Earth radii $R_{E} \simeq 6370 \mathrm{~km}$. It should be kept in mind that $L^{*}$, and therefore $\Phi$, are adiabatic invariants and that a change in $L^{*}$ along a drift orbit can result in irreversible energization of electrons. The reader unfamiliar with radial diffusion can find a Hamiltonian description of the problem in Lejosne \& Kollmann (2020). Our starting point is Equation (54) of Lejosne (2019) for the radial motion of an electron drifting around the equator of a dipolar background magnetic field:

$$
\frac{d L^{*}}{d t}=-\frac{5}{7} \frac{L^{* 5} R_{E}}{B_{E}} \frac{d A}{d t} \cos (\varphi) .
$$

We first make a change of notation by writing $\widetilde{\delta B}=R_{E} A$. The quantity $\widetilde{\delta B}$ has units of Tesla and will be treated as a continuous random variable with a zero time average $\langle\widetilde{\delta B}\rangle=$ $\frac{1}{T} \int_{0}^{T} \widetilde{\delta B} d t=0,{ }^{7}$ and finite variance $\left\langle\widetilde{\delta B}^{2}\right\rangle=\frac{1}{T} \int_{0}^{T} \widetilde{\delta B}^{2} d t<\infty$ The equation of motion we will use can now be written as

$$
\frac{d L^{*}}{d t}=-\frac{5}{7} L^{* 5} \frac{d}{d t}\left(\frac{\widetilde{\delta B}}{B_{E}}\right) \cos (\varphi) .
$$

Assuming small distortions in the particle trajectory as it interacts with the fluctuations, we write $L^{*}=L_{0}^{*}+\delta L^{*}$ and $L^{* 5} \simeq L_{0}^{* 5}\left(1+5 \frac{\delta L^{*}}{L_{0}^{*}}\right)$. We also assume that the azimuthal drift is uniform, i.e., independent on local time. This assumption can

\footnotetext{
6 A more sophisticated model for the fluctuations could also be taken into account. However, Schulz \& Eviatar (1969) showed that our Equation (3) is a good first approximation to analyze radial diffusion. They found that in the case of a slightly asymmetric background field, the value of the radial diffusion coefficient is proportional to the power spectrum of the field fluctuations at all harmonics of the drift frequency, although the first harmonic remains the main contributor.

7 On the basis of our assumption of stationarity, the mean is constant, and independent of time. However, observationally, the mean is not necessarily 0 since the magnetic field is always a bit stretched, $\langle A(t)\rangle \neq 0$, and does not necessarily average to a dipole. In that case, $\langle\delta B\rangle=R_{E}(A(t)-\langle A(t)\rangle)$, and the formulas provided below are still valid.
} 
be written as $\varphi(t)=\varphi_{0}+\Omega_{D} t .^{8}$ Using these assumptions we seek to compute the increment $\Delta L^{*}$ and dispersion $\Delta L^{* 2}$ for an ensemble of particles with initial conditions $L^{*}(t=0)=L_{0}^{*}$ and $\varphi(t=0)=\varphi_{0}$. We first determine the drift in terms of the fluctuation amplitude of the signal $\widetilde{\delta B}$, instead of its derivative. ${ }^{9}$ Integrating by part, we find

$$
\begin{aligned}
& \Delta L^{*}(t)=-\frac{5}{7} \frac{L^{* 5}}{B_{E}}\left[\widetilde{\delta B}(t) \cos \left(\Omega_{D} t+\varphi_{0}\right)\right. \\
& \left.-\widetilde{\delta B}(0) \cos \left(\varphi_{0}\right)+\Omega_{D} \int_{0}^{t} \widetilde{\delta B}(u) \sin \left(\Omega_{D} u+\varphi_{0}\right) d u\right] .
\end{aligned}
$$

Taking the square of Equation (5) and performing the ensemble average over the initial phase $\varphi_{0}$, we find

$$
\begin{aligned}
\left\langle\Delta L^{* 2}\right\rangle_{\varphi}= & \frac{25}{49} \frac{L^{* 10}}{B_{E}^{2}}\left[\frac{1}{2} \widetilde{\delta B}^{2}(t)\right. \\
& +\frac{1}{2} \widetilde{\delta B}^{2}(0)-\widetilde{\delta B}(t) \widetilde{\delta B}(0) \cos \left(\Omega_{D} t\right) \\
& +\Omega_{D} \int_{0}^{t} \widetilde{\delta B}(t) \widetilde{\delta B}(u) \sin \left(\Omega_{D}(u-t)\right) d u \\
& -\Omega_{D} \int_{0}^{t} \widetilde{\delta B}(u) \widetilde{\delta B}(0) \sin \left(\Omega_{D} u\right) d u \\
& \left.+\frac{\Omega_{D}^{2}}{2} \int_{0}^{t} \int_{0}^{t} \widetilde{\delta B}(u) \widetilde{\delta B}(v) \cos \left(\Omega_{D}(u-v)\right) d u d v\right] .
\end{aligned}
$$

We distinguish time averages from averages over the phase with as follows: $\langle X\rangle_{\varphi_{0}}$ denotes an average over the phase $\varphi_{0}$, and $\langle X\rangle_{\varphi, \widetilde{\delta B}}$ an ensemble average over the phase followed by a time average over the fluctuation $\widetilde{\delta B}$. If we now assume time homogeneous fluctuations, ${ }^{10}$ we find the following integral:

$$
\begin{aligned}
\left\langle\Delta L^{* 2}\right\rangle_{\varphi, \widetilde{B B}}= & \frac{25}{49} \frac{L^{* 10}}{B_{E}^{2}}\left[\left\langle\widetilde{\delta B} \widetilde{B B}^{2}\right\rangle-\langle\widetilde{\delta B}(t) \widetilde{\delta B}(0)\rangle \cos \left(\Omega_{D} t\right)\right. \\
& +\Omega_{D} \int_{0}^{t}\langle\widetilde{\delta B}(t) \widetilde{\delta B}(u)\rangle \sin \left(\Omega_{D}(u-t)\right) d u \\
& -\Omega_{D} \int_{0}^{t}\langle\widetilde{\delta B}(u) \widetilde{\delta B}(0)\rangle \sin \left(\Omega_{D} u\right) d u+\frac{\Omega_{D}^{2}}{2} \\
& \left.\int_{0}^{t} \int_{0}^{t}\langle\widetilde{\delta B}(u) \widetilde{\delta B}(v)\rangle \cos \left(\Omega_{D}(u-v)\right) d u d v\right] .
\end{aligned}
$$

\footnotetext{
8 In the case of a simplified Mead field, the increment in the azimuthal phase is written in terms of the first adiabatic invariant $\mu$ and symmetric and antisymmetric fluctuations, $S(t)$ and $A(t)$, respectively: $\Delta \varphi(t)=$ $-\frac{3 \mu}{\gamma q r^{2}} t+\int^{t}\left(\frac{3 \mu S(\tau) r}{\gamma q B_{E} R_{E}^{3}}+4 \frac{\mu A(\tau) r^{2}}{\gamma q B_{E} R_{E}^{3}} \cos (\varphi)+\frac{1}{7} \frac{r^{4}}{B_{E} R_{E}^{3}} \frac{d A}{d \tau} \sin (\varphi)\right) d \tau$. It is typically considered that $\Omega_{D}=-\frac{3 \mu}{a^{2}}$, but it is clear that the Alfvénic fluctuations can affect the angular drift frequency.

9 We can do the same analysis for the time derivative of the signal, i.e., $d A / d t$, but it is preferable to determine the transport coefficients in terms of the magnetic field fluctuations $A(t)$ since it can be implemented in terms of magnetopause location and its statistical properties obtained from in situ measurements (Schulz \& Eviatar 1969). Observationally, it is also unnecessarily arduous to extract the Lagrangian time derivative of the signal, when $A(t)$ and its associated statistical properties suffice.

${ }^{10}$ That is, stationarity in the sense that the autocorrelation function of the fluctuations $\left\langle\widetilde{\delta B}\left(t_{1}\right) \widetilde{\delta B}\left(t_{2}\right)\right\rangle$ depends solely on the lag $\tau=t_{2}-t_{1}$.
}

Making a change of variables in the first integrals, and taking advantage of the stationarity of the signal in the double integral, one finds

$$
\begin{aligned}
\left\langle\Delta L^{* 2}\right\rangle_{\varphi, \widetilde{\delta B}}= & \frac{25}{49} \frac{L^{* 10}}{B_{E}^{2}}\left[\left\langle\widetilde{\delta B}^{2}\right\rangle-\langle\widetilde{\delta B}(t) \widetilde{\delta B}(0)\rangle \cos \left(\Omega_{D} t\right)\right. \\
& -2 \Omega_{D} \int_{0}^{t}\langle\widetilde{\delta B}(0) \widetilde{\delta B}(u)\rangle \sin \left(\Omega_{D} u\right) d u \\
& \left.+\Omega_{D}^{2} t \int_{0}^{t}\langle\widetilde{\delta B}(u) \widetilde{\delta B}(0)\rangle \cos \left(\Omega_{D} u\right) d u\right]
\end{aligned}
$$

For a given form of the autocorrelation function we can compute the time-dependent diffusion coefficient $D=\frac{1}{2} \frac{d}{d t}\left\langle\Delta L^{* 2}\right\rangle_{\varphi_{0}, \widetilde{B B}}$ (Taylor 1922). If the covariance of the fluctuations has zero correlation time, we can set the limit of the integral to $t=\infty$, the autocorrelation function to a delta function $\langle\widetilde{\delta B}(0) \widetilde{\delta B}(\tau)\rangle=$ $\left\langle\widetilde{\delta B}^{2}\right\rangle \delta\left(\tau / \tau_{c}\right)$, and recover the traditional linear time dependence of the dispersion (Taylor 1922): $\left\langle\Delta L^{* 2}\right\rangle_{\varphi_{0}, \widetilde{B B}}=\frac{25}{49} L^{* 10} \Omega_{D}^{2} \tau_{c} \frac{\left\langle\widetilde{\delta B}^{2}\right\rangle}{B_{E}^{2}} t$.

\subsection{Incorporating an Autocorrelation Function Time}

We now introduce a conceptually simple autocorrelation function with finite autocorrelation time $\tau_{c}:{ }^{11}$

$$
\langle\widetilde{\delta B}(0) \widetilde{\delta B}(\tau)\rangle=\left\langle\widetilde{\delta B}^{2}\right\rangle e^{-\frac{\tau}{\tau_{c}}}
$$

The parameter $\tau_{c}$ is a constant and denotes the characteristic time for fluctuations to decorrelate. Inserting the autocorrelation in Equation (9) into Equation (8) we can solve the integral analytically:

$$
\begin{aligned}
& \left\langle\Delta L^{* 2}\right\rangle_{\varphi_{0}, \widetilde{B B}}=\frac{25}{49} L^{* 10} \frac{\left\langle\widetilde{\delta B}^{2}\right\rangle}{B_{E}^{2}}\left[1-e^{-\frac{t}{\tau_{c}}} \cos \left(\Omega_{D} t\right)\right. \\
& +\frac{2 \Omega_{d} \tau_{c}}{\Omega_{D}^{2} \tau_{c}^{2}+1}\left(e^{-\frac{t}{\tau_{c}}} \Omega_{D} \tau_{c} \cos \left(\Omega_{D} t\right)+e^{-\frac{t}{\tau_{c}}} \sin \left(\Omega_{D} t\right)-\Omega_{D} \tau_{c}\right) \\
& \left.+\frac{\Omega_{D}^{2} t \tau_{c}}{\Omega_{D}^{2} \tau_{c}^{2}+1}\left(1-e^{-\frac{t}{\tau_{c}}} \cos \left(\Omega_{D} t\right)+e^{-\frac{t}{\tau_{c}}} \Omega_{D} \tau_{c} \sin \left(\Omega_{D} t\right)\right)\right] .
\end{aligned}
$$

In the limit $t<\tau_{c} \ll \tau_{L} \simeq \Omega_{D}^{-1}$ the dispersion of an ensemble of particles is linear in time:

$$
\left\langle\Delta L^{* 2}\right\rangle_{\varphi_{0}, \widetilde{B B}}=\frac{25}{49} L^{* 10} \frac{\left\langle\left\langle\widetilde{\delta B}^{2}\right\rangle\right.}{B_{E}^{2}} \frac{t}{\tau_{c}} .
$$

However, the introduction of finite correlation times leads to transient oscillatory dispersion if $\tau_{L}<t \leqslant \tau_{c}$ and linear dispersion if $t \gg \tau_{c}$ :

$$
\left\langle\Delta L^{* 2}\right\rangle_{\varphi_{0}, \widetilde{B B}}=\frac{25}{49} L^{* 10} \frac{\left\langle\widetilde{\delta B}^{2}\right\rangle}{B_{E}^{2}} \frac{\Omega_{D}^{2} t \tau_{c}}{1+\tau_{c}^{2} \Omega_{D}^{2}} .
$$

\footnotetext{
${ }^{11}$ Lejosne et al. (2013) estimate empirically the autocorrelation function as a sum of two exponentials with parameters dependent on the $K_{p}$ index. Our choice of an exponential autocorrelation function is coherent with previous empirical estimates and our results can easily be extrapolated to a sum of two exponentials composed of both short and long autocorrelation times.
} 


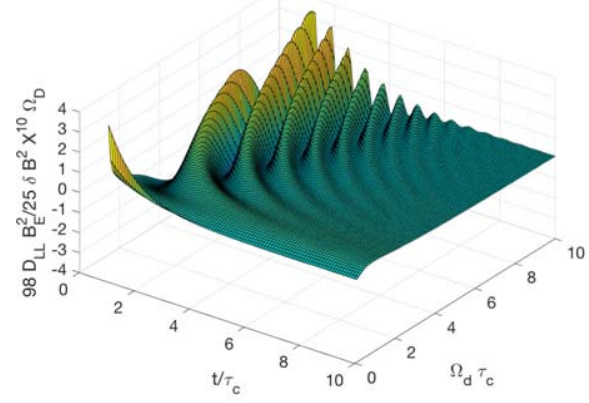

(a)

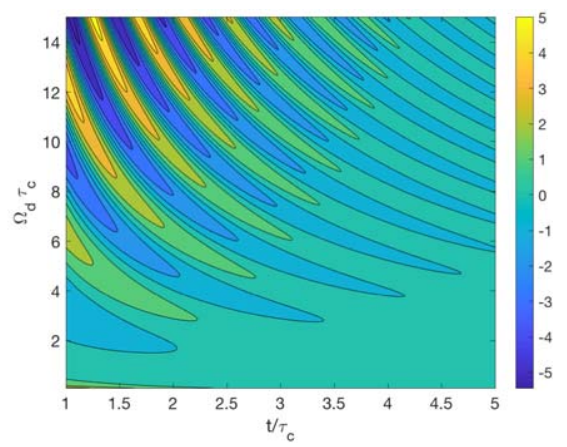

(b)

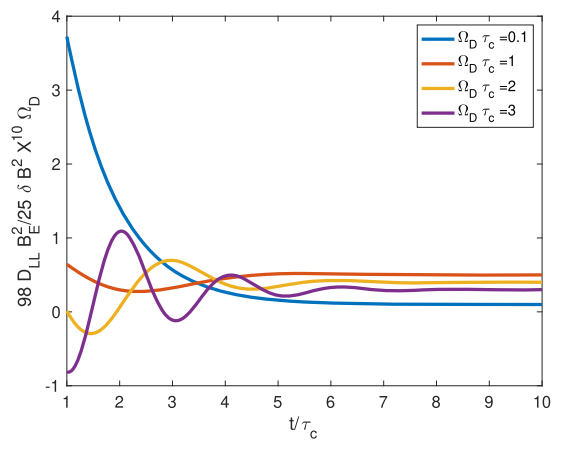

(c)

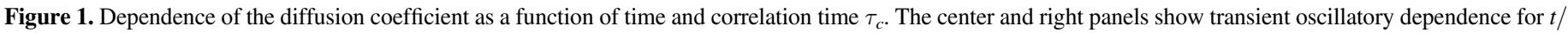
$\tau_{c} \leqslant 5$ and the convergence to the steady values for $t \gg \tau_{c}$.

Computing the time-dependent radial diffusion coefficient $D_{L L}=\frac{1}{2} \frac{d}{d t}\left\langle\Delta L^{* 2}\right\rangle_{\varphi_{0}}, \widetilde{\delta B}$ from Equation (10) we find

$$
\begin{aligned}
D_{L L}= & \frac{25}{98} \frac{\left\langle\widetilde{\delta B}^{2}\right\rangle}{B_{E}^{2}} L^{* 10} \Omega_{D}\left[\frac{\Omega_{D} \tau_{c}}{1+\tau_{c}^{2} \Omega_{D}^{2}}+e^{-\frac{t}{\tau_{c}}} \cos \left(\Omega_{D} t\right)\right. \\
& \times\left(\frac{1}{\Omega_{D} \tau_{c}}+\Omega_{D} t-\frac{\Omega_{D} \tau_{c}}{1+\Omega_{D}^{2} \tau_{c}^{2}}\right)-e^{-\frac{t}{\tau_{c}}} \sin \left(\Omega_{D} t\right) \\
& \left.\times\left(1-\frac{\Omega_{D}^{2} \tau_{c}^{2}}{1+\Omega_{D}^{2} \tau_{c}^{2}}\right)\right] .
\end{aligned}
$$

Similarly as for dispersion, the introduction of finite correlation times leads to transient oscillatory diffusion for an ensemble of particles. The time dependence of the function in brackets in Equation (13) is shown in Figure 1. For $t / \tau_{c} \simeq 1$ and $\Omega_{D} \tau_{c}>1$, the diffusion coefficient has an oscillatory time dependence with period $\Omega_{D}^{-1}$. But as $t \geqslant_{c} \gg \tau_{c}$ the diffusion coefficient takes the following time-independent form:

$$
D_{L L}\left(t \gg \tau_{c}\right)=\frac{25}{98} L^{* 10} \frac{\left\langle\widetilde{\delta B}^{2}\right\rangle}{B_{E}^{2}} \frac{\Omega_{D}^{2} \tau_{c}}{1+\tau_{c}^{2} \Omega_{D}^{2}} .
$$

\subsection{Equilibrium Distribution Function along $\mathrm{L}^{*}$ for Finite Correlation Time $\tau_{\mathrm{c}} \neq 0$}

We now seek equilibrium solutions with the diffusion coefficient that incorporates the effect of finite correlation times $\tau_{c} \neq 0$, in the limit where $t \gg \tau_{c}$ for the the diffusion coefficient in Equation (14). To find the most general solution, let us assume a linear dependence of the drift frequency $\Omega_{D}=\beta L^{-2},{ }^{12}$ an assumption that is realistic in a mean dipole field with magnetic drifts dominating over the electric drifts. The coefficient $\beta(\mu, \mathcal{E})$ has units of $\left(s^{-1}\right)$ and has a dependence on the first adiabatic invariant and the energy of the electron $\mathcal{E}=\gamma m_{e} c^{2}$. The Fokker-Planck equation describing the time-dependent evolution of an ensemble of particles along $L$ can be written as (Lichtenberg \& Lieberman 1983)

$$
\frac{\partial f}{\partial t}=\frac{\partial}{\partial L}\left(\frac{D_{L L}}{L^{2}} \frac{\partial}{\partial L}\left(f L^{2}\right)\right)
$$

\footnotetext{
${ }^{12}$ To simplify the notation we now write $L^{*} \rightarrow L$.
}

We will seek a steady-state solution for the function $g(L)=L^{2} f$, i.e.,

$$
\frac{d}{d L}\left(\frac{D_{L L}}{L^{2}} \frac{d g}{d L}\right)=0 .
$$

The solution to this equation can be written in terms of the constants $c_{1}$ and $c_{2}$, determined with the choice of boundary conditions $g\left(L=L_{\min }\right)=0$ and $g\left(L=L_{\max }\right)=g_{m}$ as

$$
g\left(L, t \gg \tau_{c}\right)=c_{1}+\frac{c_{2}}{L^{7}}\left(3+\frac{7}{\Omega_{D}^{2} \tau_{c}^{2}}\right) .
$$

Thus, the equilibrium distribution is independent of the rms magnetic fluctuation and depends solely on $L$ and $\Omega_{D} \tau_{c}$. In order to characterize the dependence of the equilibrium distribution in terms of the parameter $\beta \tau_{c}$ we can set the boundary conditions at $L_{\min }=1$ and $L_{\max }=8$ with $g_{m}=1$. The inner boundary condition indicates that particles beyond $L=1$ are efficiently lost to the neutral atmosphere, and the outer boundary assumes a steady value corresponding to the magnetopause boundary on the dayside of the magnetosphere. Using the above solution we can determine the effect of the parameterized finite correlation times $\beta \tau_{c}$ on the equilibrium distribution. The results are shown in Figure 2 for $g(L)$ (panel a) and $f(L)$ (panel b). We note that the shape of the equilibrium distribution is not affected by the finite correlation time. However for very short (long) correlation times $\beta \tau_{c} \ll 1$ $\left(\beta \tau_{c} \gg 1\right)$ the distribution $g(L)$ declines at a higher (lower) $L$ but values of $\beta \tau_{c}$ account for differences of at most ten percents for $L^{*}<3$ and of less than a percent for $L^{*}>4$. This dependence of the phase-space density on the parameter $\beta \tau_{c}$ for fixed values of $L=[2,2.5,3,3.5]$ is more clearly shown on panel (c) of Figure (2). Thus, finite correlation times do not significantly affect the long term equilibrium distribution but longer autocorrelation times result in a more efficient filling of the inner belts. In the next sections we will seek solutions of the Fokker-Planck equation on intermediate timescales.

\subsection{Time-dependent Evolution of the Distribution Function with $\tau_{\mathrm{c}} \neq 0$}

In this section we seek self-similar asymptotic solutions of the time-dependent Equation (15) derived in the limit $t \gg \tau_{c}$. Our aim is to determine the time dependence of the variance along the third 


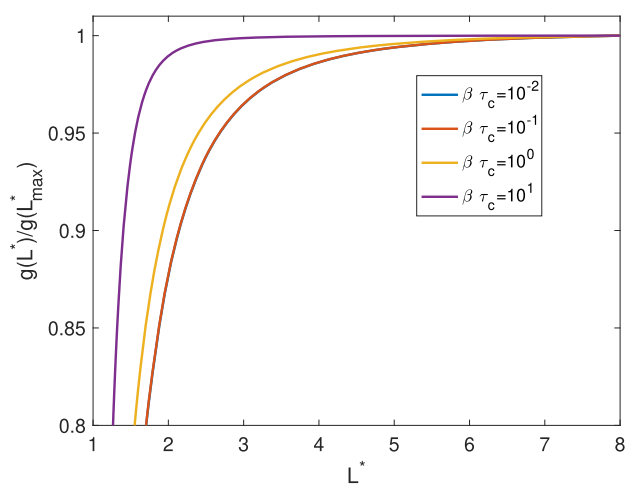

(a)

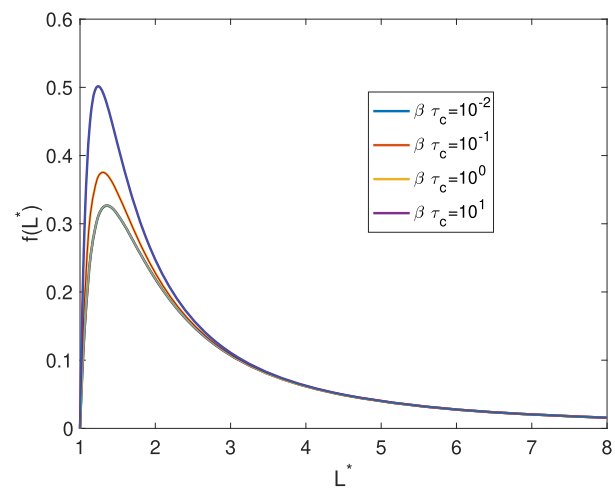

(b)

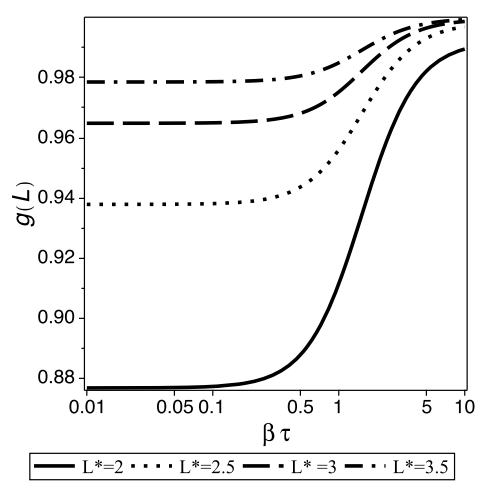

(c)

Figure 2. Panels (a) and (b) show the equilibrium distribution function $g\left(L^{*}\right)$ and $f=g / L^{* 2}$ as a function of $L^{*}$ for normalized autocorrelation times $\beta(\mu, \mathcal{E}) \tau_{c}$ ranging between $10^{-2}$ and $10^{2}$. In panel (c) the phase-space density for fixed values $L^{*}=[2,2.5,3,3.5]$ is plotted as a function of the parameter $\beta \tau_{c}$.

adiabatic invariant, i.e., $\left\langle\Delta L^{* 2}\right\rangle \sim t^{s}$, and therefore estimate whether the transport of particles is diffusive $(s=1)$ or not $(s \neq 1)$. A quantitative description of the diffusion for short and long autocorrelation time allows us to use a radiation belt model in order to extract information of the fluctuations responsible for the transport of electrons. Self-similar solutions describe intermediate asymptotic behavior of solutions in the region where the solution is no longer dependent on the details of the initial and boundary conditions but the system is still far from being in a state of equilibrium (Barenblatt 1996). By assuming an open external boundary, i.e., $L^{*} \in[0, \infty]$, it is shown that self-similar solutions exist in the limits of small but finite autocorrelation times (i.e., $\tau_{c} \Omega_{d} \ll 1$ ) and long autocorrelation time (i.e., $\tau_{c} \Omega_{d} \gg 1$ ) and allow us to quantify the diffusion rate under each respective regime.

\subsubsection{Self-similar Solutions for $\Omega_{\mathrm{d}} \tau_{\mathrm{c}} \gg 1$}

Our starting point is the diffusion Equation (15) written in terms of $g\left(L^{*}\right)=f L^{* 2}$ and the inverse of $L^{*}-1=\chi$ :

$$
\frac{\partial g}{\partial t}=\frac{\partial}{\partial \chi}\left(\chi^{4} D_{L L} \frac{\partial g}{\partial \chi}\right) .
$$

In the limit of long autocorrelation times $\left(\tau_{c} \gg \Omega_{d}^{-1}\right)$ the diffusion coefficient (14) can be approximated as

$$
D_{L L} \simeq \frac{25}{98} \frac{\left\langle\widetilde{\delta B}^{2}\right\rangle}{B_{E}^{2}} \frac{1}{\tau_{c}} \chi^{-10}
$$

and the diffusion Equation (18) for magnetic field autocorrelation $\left\langle\widetilde{\delta B}^{2}\right\rangle$ independent of $L^{*}$ is written as

$$
\frac{\partial g}{\partial t}=\frac{25}{98} \frac{\left\langle\widetilde{\delta B}^{2}\right\rangle}{B_{E}^{2}} \frac{1}{\tau_{c}} \frac{\partial}{\partial \chi}\left(\frac{1}{\chi^{6}} \frac{\partial g}{\partial \chi}\right) .
$$

Normalizing time $\tau=\sigma t$ with the parameter $\sigma=\frac{25}{98} \frac{\left\langle\widetilde{\delta B}^{2}\right\rangle}{B_{E}^{2}} \tau_{c}^{-1}$ the diffusion equation can be written compactly as

$$
\frac{\partial g}{\partial \tau}=\frac{\partial}{\partial \chi}\left(\frac{1}{\chi^{6}} \frac{\partial g}{\partial \chi}\right)
$$

By dimensional analysis, a self-similar solution can be found in terms of the function $g_{>}=\Psi(\kappa) / \tau^{\lambda}{ }^{13}$ for the variable $\kappa=\chi^{8} / \tau$ and the constant to be determined $\lambda$. This change of variable from $(\chi, \tau) \rightarrow(\kappa, \tau)$ reduces the partial differential Equation (21) into the following ordinary differential equation:

$$
64 \kappa \Psi^{\prime \prime}+(8+\kappa) \Psi^{\prime}+\lambda \Psi=0 .
$$

In the absence of sources and sinks, we require that the phasespace density along $\chi$, or equivalently $L^{*}$, be conserved for the domain $L^{*} \in\left[0, L_{\max }^{*}\right]$, or equivalently $\chi \in\left[L_{\max }^{*-1}, \infty\right]$. The asymptotic limit in which our solution is valid is detailed below in terms of the number density of particles $n_{s}$. This translates into the following condition:

$$
\int_{L_{\max }^{-1}}^{\infty} g_{>}(\chi, t) d \chi=n_{s}
$$

Written in terms of the variable $\kappa$, the integral takes the following form:

$$
\frac{1}{8} \frac{1}{\tau^{\lambda-1 / 8}} \int_{\tau^{-1} L_{\max }^{-8}}^{\infty} \Psi(\kappa) \kappa^{-7 / 8} d \kappa=n_{s} .
$$

In order for the number of particles to be constant in time since we have no sink nor source, we require our self-similar solution to have $\lambda=1 / 8$ and the lower bound of the integral to always remain small, i.e., $\varepsilon=\tau^{-1} L_{\max }^{-8} \ll 1$. The second-order ODE in Equation (22) can then be written in terms $P=\Psi^{\prime}+\Psi / 64$ as

$$
\kappa P^{\prime}+\frac{1}{8} P=0
$$

with the solution $P=c_{1} \kappa^{-1 / 8}$ and integration constant $c_{1}$. The general solution for Equation (22) can be written as

$$
\Psi(\kappa)=e^{-\kappa / 64}\left(c_{2}+c_{1} \int_{-\infty}^{\kappa} \kappa^{\prime-1 / 8} e^{\kappa^{\prime} / 64} d \kappa^{\prime}\right) .
$$

Since the solution needs to be finite to conserve the number of particles under the constraint (24), we set $c_{1}=0 .{ }^{14}$ Using this

\footnotetext{
${ }^{13}$ The subscript symbol $>$ indicates that we are looking at a solution for the long autocorrelation time. In the next section, the distribution function for the short autocorrelation time will be denoted with the subscript $<$, i.e., $g_{<\text {. }}$

${ }^{14}$ Inserting Equation (26) into Equation (24) results in a logarithmic divergent integral $\int_{0}^{\infty} \kappa^{-1} d \kappa$, so we have to set $c_{1}=0$.
} 

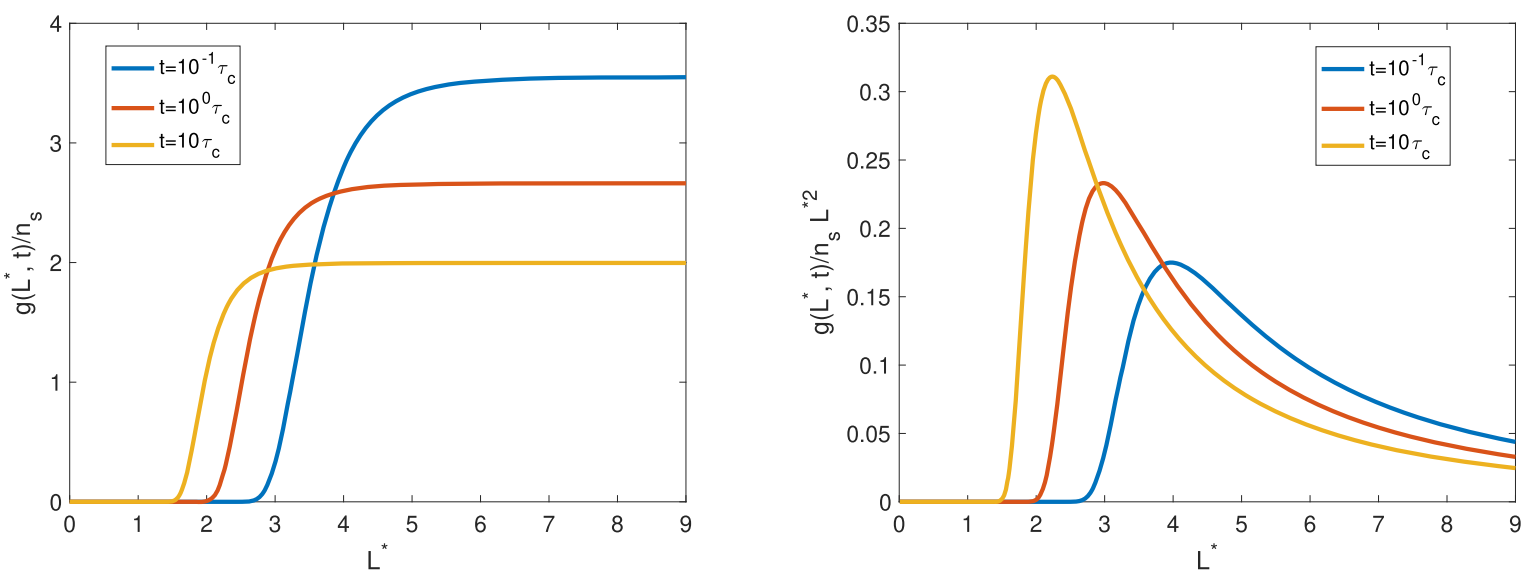

Figure 3. Evolution of the distribution function $g\left(L^{*}, t\right)$ and $f=g / L^{* 2}$ as a function of $L^{*}$ and $t$ for long autocorrelation times $\tau_{c} \gg \Omega_{D}^{-1}$. The rms magnetic fluctuation is set to $\sqrt{\left\langle\delta B^{2}\right\rangle}=0.01 B_{E}$.

same constraint, i.e., integral (24), we can find the value of $c_{2}$ and the general solution for $g\left(\chi=1 / L^{*}, t\right)$ in terms of the gamma function $\Gamma(z)=\int_{0}^{\infty} x^{z-1} e^{-x} d x$ for $\mathfrak{R}(z)>0$ and the identity $\Gamma(z) \Gamma(1-z)=\pi / \sin (\pi z)$ :

$$
g_{>}\left(L^{*}, t\right)=\frac{8^{3 / 4} n_{s}}{\Gamma\left(\frac{1}{8}\right)(\sigma t)^{1 / 8}} e^{-\frac{1}{64 L^{* 8 \sigma t}}} .
$$

The solutions $g_{>}\left(L^{*}, t\right)$ and $f_{>}\left(L^{*}, t\right)=g_{>} / L^{* 2}$ are plotted in Figure 3 to highlight the flattening of phase-space density with time. It should be kept in mind that in order to be consistent with the lower bound integral limits in Equations (23) and (24), this solution is valid for timescales:

$$
\Omega_{D} t \gg 3.92 \frac{\left(\Omega_{D} \tau_{c}\right)_{>}}{L_{\max }^{8}} \frac{B_{E}^{2}}{\left\langle\widetilde{\delta B}^{2}\right\rangle}
$$

with the notation $\left(\Omega_{D} \tau_{c}\right)_{>}$indicating that the term is much larger than 1 and corresponds to the value for long autocorrelation time. Even though our solution is only valid in the asymptotic limit defined above, it allows us to infer that the diffusion along $\chi$ or $L^{*}$ is subdiffusive and independent of the drift frequency $\Omega_{D}$. The subdiffusive transport for the long autocorrelation time can be computed exactly from the variance in $\chi$ according to

$$
\begin{aligned}
\left\langle\Delta \chi^{2}\right\rangle_{>} & =\left\langle\chi^{2}\right\rangle_{>}-\langle\chi\rangle_{>}^{2} \\
& =\int_{0}^{\infty} \chi^{2} g_{>}(\chi, t) d \chi-\left(\int_{0}^{\infty} \chi g_{>}(\chi, t) d \chi\right)^{2} \\
& =2^{3 / 2} n_{s}(\sigma t)^{1 / 4} \frac{\Gamma(3 / 8)}{\Gamma(1 / 8)}\left(1-n_{s} \frac{\Gamma(1 / 4)^{2}}{\Gamma(1 / 8) \Gamma(3 / 8)}\right) \\
& \simeq 2^{3 / 2} n_{s} \frac{\Gamma(3 / 8)}{\Gamma(1 / 8)}(\sigma t)^{1 / 4}
\end{aligned}
$$

Thus, the square root of the variance in $\chi$ scales as $\sqrt{\left\langle\Delta \chi^{2}\right\rangle} \sim t^{1 / 8}$, instead of $\sqrt{\left\langle\Delta \chi^{2}\right\rangle} \sim t^{1 / 2}$ as would be expected for standard diffusion.

\subsubsection{Self-similar Solutions for $\Omega_{\mathrm{d}} \tau_{\mathrm{c}} \ll 1$}

In the limit of short autocorrelation times $\left(\tau_{c} \ll \Omega_{d}^{-1}\right)$ the diffusion coefficient (14) can be approximated as

$$
D_{L L} \simeq \frac{25}{98} \frac{\left\langle\widetilde{\delta B}^{2}\right\rangle}{B_{E}^{2}} \Omega_{d}^{2} \tau_{c} \chi^{-10},
$$

and the diffusion Equation (18) as

$$
\frac{\partial g}{\partial t}=\frac{25}{98} \frac{\left\langle\widetilde{\delta B}^{2}\right\rangle}{B_{E}^{2}} \frac{1}{\tau_{c}}\left[\frac{\partial}{\partial \chi}\left(\frac{\Omega_{d}^{2} \tau_{c}^{2}}{\chi^{6}} \frac{\partial g}{\partial \chi}\right)\right] .
$$

Normalizing time $\tau=\sigma t$ with the parameter $\sigma=\frac{25}{98} \frac{\left\langle\widetilde{\delta B}^{2}\right\rangle}{B_{E}^{2}} \tau_{c}^{-1}$ and assuming a dependence of the drift frequency $\Omega_{d}=\beta L^{-2}=\beta \chi^{2}$ the diffusion equation can be written compactly as

$$
\frac{1}{\beta^{2} \tau_{c}^{2}} \frac{\partial g}{\partial \tau}=\frac{\partial}{\partial \chi}\left(\frac{1}{\chi^{2}} \frac{\partial g}{\partial \chi}\right)
$$

Following the exact same procedure as in the previous section, a self-similar solution can be found in terms of the function $g_{<}=\Psi(\kappa) / \tau^{\lambda}$ for the variable $\kappa=\chi^{4} / \tau$. The second-order ODE that needs to be solved can then be written in terms $P=\Psi^{\prime}+\Psi / 16$ and $\kappa P^{\prime}+P / 4=0$. By enforcing the constraint (23), it can be shown that time-dependent solutions for small autocorrelation times can be written as

$$
g_{<}\left(L^{*}, t\right)=\frac{\sqrt{2}}{\pi} \frac{n_{s} \Gamma(3 / 4)}{(\sigma t)^{1 / 4}\left(\beta \tau_{c}\right)_{<}^{1 / 2}} e^{-\frac{1}{16 L^{* 4}\left(\beta \tau_{c}\right)^{2}<t}} .
$$

The flattening of $g_{<}\left(L^{*}, t\right)$ and the spread $f_{<}\left(L^{*}, t\right)=g_{<} / L^{* 2}$ across lower $L^{*}$ are shown in Figure 4. The above solution is valid in the limit of

$$
\beta t \gg 3.92 \frac{1}{L_{\max }^{4}} \frac{B_{E}^{2}}{\left\langle\widetilde{\delta B}^{2}\right\rangle} \frac{1}{\left(\beta \tau_{c}\right)_{<}},
$$

or written in terms of the drift frequency as

$$
\Omega_{D} t \gg 3.92 \frac{1}{L^{4} L_{\max }^{4}} \frac{B_{E}^{2}}{\left\langle\delta \widetilde{B B}^{2}\right\rangle} \frac{1}{\left(\Omega_{D} \tau_{c}\right)_{<}} .
$$

We note from the temporal dependence in denominator that the diffusion along $\chi$ for particles interacting with fluctuations that 

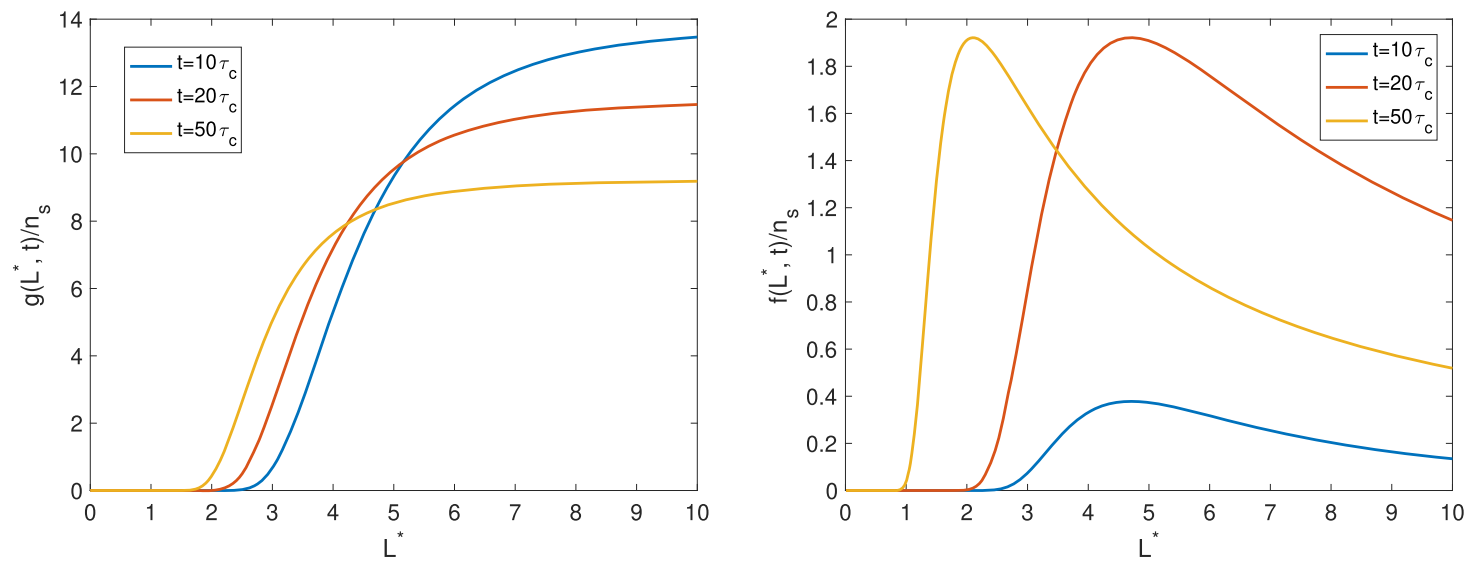

Figure 4. Evolution of the distribution function $g\left(L^{*}, t\right)$ and $f=g / L^{* 2}$ as a function of $L^{*}$ and $t$ for short autocorrelation times $\tau_{c} \ll \Omega_{D}^{-1}$. The rms magnetic fluctuation is set to $\sqrt{\left\langle\delta B^{2}\right\rangle}=0.01 B_{E}$ and $\Omega_{D} \tau_{c} L^{* 2}=\beta \tau_{c}=0.1$.

have very short autocorrelation time is also subdiffusive but dependent on the drift period through the term $\beta$. Similarly to the case for long autocorrelation time, we compute the variance in $\chi$ to find

$$
\begin{aligned}
\left\langle\Delta \chi^{2}\right\rangle_{<} & =\left\langle\chi^{2}\right\rangle_{<}-\langle\chi\rangle_{<}^{2} \\
= & \int_{0}^{\infty} \chi^{2} g_{<}(\chi, t) d \chi-\left(\int_{0}^{\infty} \chi g_{<}(\chi, t) d \chi\right)^{2} \\
= & \frac{2^{3 / 2}}{\pi} n_{s} \Gamma(3 / 4)^{2} \beta \tau_{c}(\sigma t)^{1 / 2}\left(1-\frac{n_{s}}{\sqrt{2}}\right) \\
& \simeq 1.35 n_{s}(\sigma t)^{1 / 2} L^{* 2}\left(\Omega_{D} \tau_{c}\right)_{<} .
\end{aligned}
$$

Thus, the square root of the variance in $\chi$ scales as $\sqrt{\left\langle\Delta \chi^{2}\right\rangle} \sim t^{1 / 4}$, instead of $\sqrt{\left\langle\Delta \chi^{2}\right\rangle} \sim t^{1 / 2}$, as one would find for standard diffusion. However, compared to the case for long autocorrelation times, the appearance of the drift frequency $\Omega_{D}=\beta(\mu, \mathcal{E}) L^{*-2}$ indicates that diffusion depends on the adiabatic invariant and energy of the particles. Therefore, particles of various energy and pitch-angle will experience greater variations in their diffusion rate than for long autocorrelation time. ${ }^{15}$

\subsubsection{Numerical Comparison of Diffusion Time for Arbitrary Values of $\Omega_{\mathrm{d}} \tau_{\mathrm{c}}$}

At this point we can also answer the following question: in the absence of sinks or sources, do particles diffuse faster for short or long autocorrelation time? A priori the dependence of $\left\langle\Delta \chi^{2}\right\rangle<$ as $t^{1 / 2}$ compared to $t^{1 / 4}$ suggests that transport for short autocorrelation is faster. However, taking the ratio of Equation (37) with Equation (29) gives

$$
\begin{aligned}
\frac{\left\langle\Delta \chi^{2}\right\rangle_{<}}{\left\langle\Delta \chi^{2}\right\rangle_{>}}= & 1.07\left(\frac{\left\langle\delta B^{2}\right\rangle}{B_{E}^{2}}\right)^{1 / 4}\left(\frac{\tau_{c>}}{\tau_{c<}}\right)^{1 / 4} \\
& \times\left(\Omega_{D} \tau_{c<}\right)^{3 / 4}\left(\Omega_{D} t\right)^{1 / 4} L^{* 2} \simeq\left(\Omega_{D} \tau_{c<}\right)^{3 / 4} L^{* 2} .
\end{aligned}
$$

\footnotetext{
15 The reader might have noticed that even though the dispersion in $L^{*}$ given by Equation (12) is linear in time, the diffusion Equation (18) for both long and short autocorrelation times gives subdiffusive transport. In order to obtain a classical diffusive transport that scales as $\left\langle\Delta L^{* 2}\right\rangle \sim t$ the diffusion coefficient must have a dependence of $D_{L L} \sim L^{* 4}$.
}

With a ratio of long to short autocorrelation time of order $\frac{\tau_{c>}}{\tau_{c<}} \simeq 30$ (Lejosne et al. 2013) the first four terms on the first line are all comparable or smaller than 1 and the above coefficient is much smaller than unity even for $\Omega_{D} t \gg 1$ and $L^{*} \in[1-10] .{ }^{16}$ We therefore expect transport due to long autocorrelation to be much faster than for short autocorrelation times across typical radiation belts condition. We can test the validity of this dimensional analysis numerically by seeking numerical solutions for arbitrary values of $\Omega_{D} \tau_{c}$ of the diffusion equation

$$
\frac{\partial g}{\partial t}=L^{2} \frac{\partial}{\partial L}\left(\frac{D_{L L}}{L^{2}} \frac{\partial g}{\partial L}\right), \text { for } 0<L<10, t>0,
$$

and subject to the boundary conditions $g(L=10, t)=g(L=$ $0, t)=1$ and the initial perturbation $g\left(L^{*}, t=0\right)=1+$ $0.2 \exp \left[-\left(L^{*}-L_{0}^{*}\right)^{2} / 0.5^{2}\right]$. We run the simulation for parameterized correlation time $\beta \tau_{c}=[0.1,1,10]$ and for a normalized time of $\beta t=10 .{ }^{17}$ Figures (5) and (6) show the result to the numerical integration from the left (a), center (b), and right (c) panels for $\beta \tau_{c}=[0.1,1.10]$ and a perturbation centered at $L^{*}=7$ and $L^{*}=5$, respectively. Consistent with the above dimensional analysis, a longer autocorrelation time $\left(\beta \tau_{c} \gg 1\right)$ results in a faster flattening of the distribution along $L$ than for a shorter autocorrelation time. As the parameter $\beta \tau_{c} \leqslant 1$ decreases it takes longer for the initial perturbation to spread along $L^{*}$. The flattening of the initial perturbation when $\beta \tau_{c} \sim 1$ is also faster than for $\beta \tau_{c}=0.1$. Additionally, we notice that the flattening of the distribution takes longer when the initial perturbation is centered at $L^{*}=5$, than $L^{*}=7$. In other words, radial diffusion is slower toward the inner belts. These difference can be interpreted in terms of the behavior of a single particle motion with short and long autocorrelation times. For long autocorrelation times $\tau_{c} \gg \tau_{L}$, particles of a

\footnotetext{
${ }^{16}$ For times $t$ comparable to 100 or 1000 drift period $\left(\Omega_{D} t\right)^{1 / 4}$ is comparable to 1 , whereas $\left(\Omega_{D} \tau_{c}\right)^{3 / 4} \ll 1$ and dominates the inequality.

17 The equation can be solved in a few lines on Maple with PDEtools >restart; with(PDEtools); with(plots) >DLL :=(L) $->$ sigma $* \mathrm{~L}^{6 *} \beta \tau_{c} /\left(\mathrm{L}^{-4 *} \beta^{2 *} \tau_{c}^{2}+1\right)>$ parabolic $:=\operatorname{diff}(\mathrm{u}(\mathrm{t}, \mathrm{x}), \mathrm{t})-$ $(x * x) *\left(\operatorname{diff}\left(\operatorname{DLL}(x) *(\operatorname{diff}(u(t, x), x)) / x^{2}, x\right)\right)=0>$ IBC $:=[u(0, x)=f(x), u(t, 0)=1, u(t, 10)=1]>$ pdesol $:=p d s o l v e$ (parabolic, IBC, numeric, timestep $=1 / 40$, spacestep=1/40)
} 


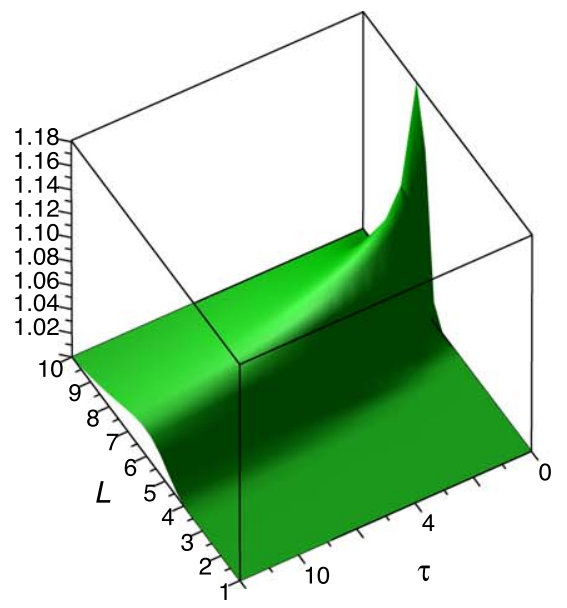

(a)

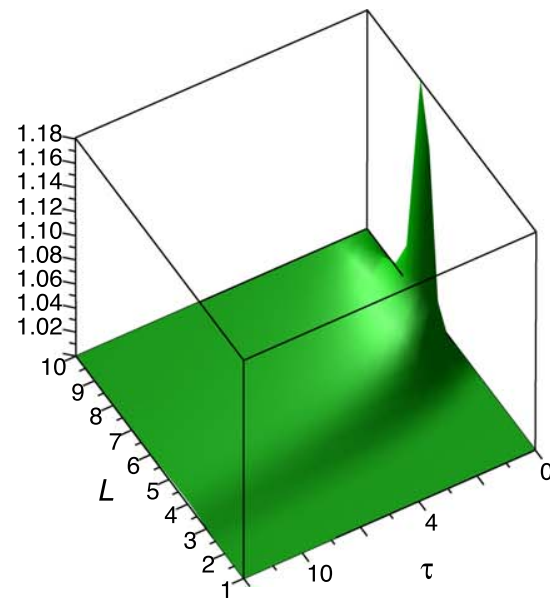

(b)

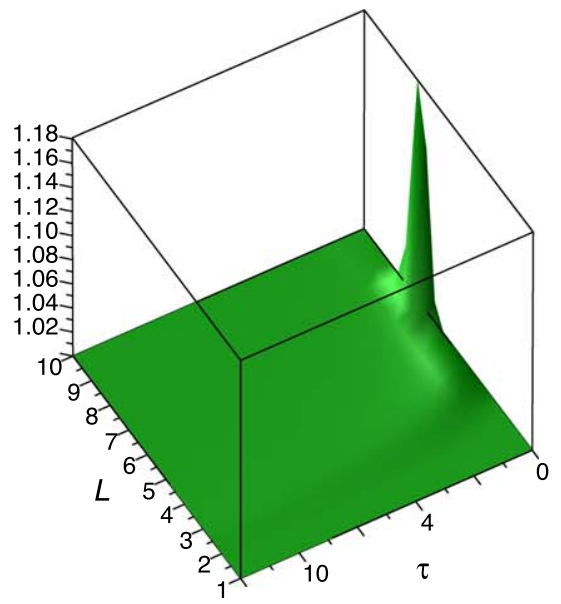

(c)

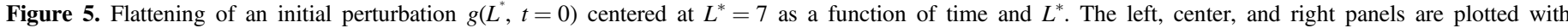

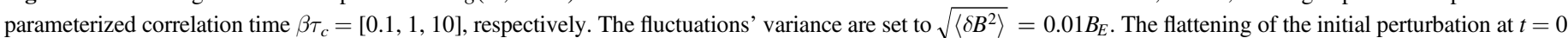
takes longer for short autocorrelation time.

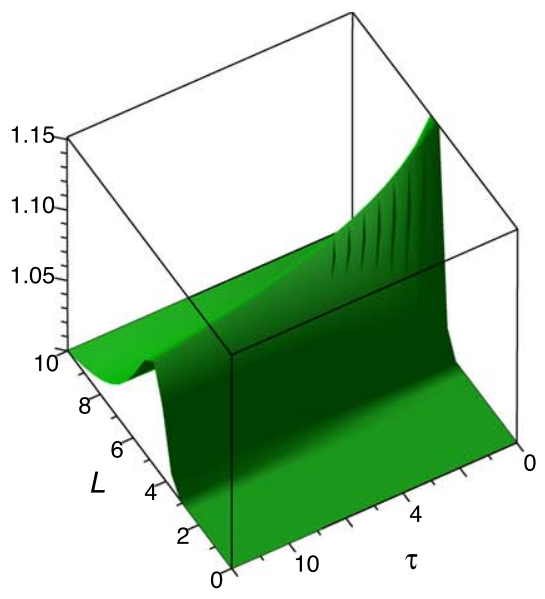

(a)

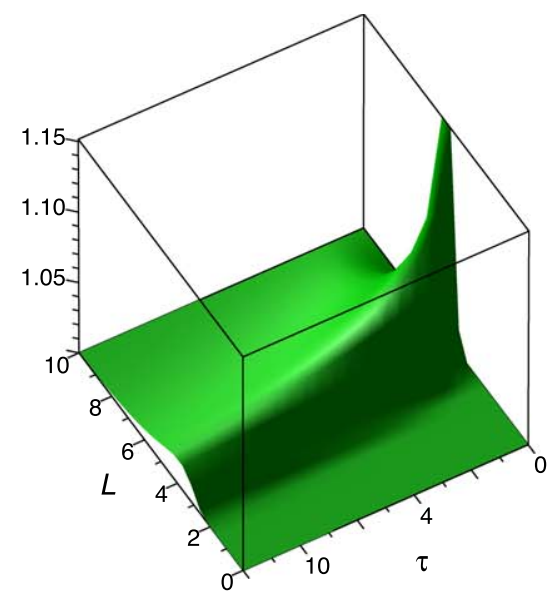

(b)

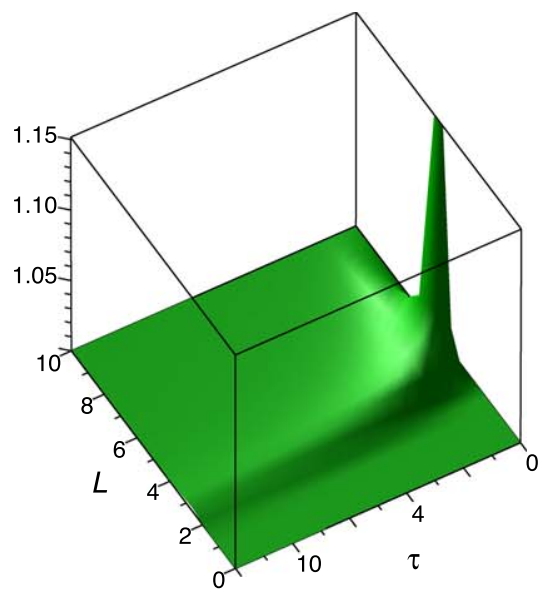

(c)

Figure 6. Same legend as in Figure 5, but with an initial perturbation centered at $L^{*}=5$.

wide range of drift frequencies will sample the antisymmetric field independently of their drift period. However, for very small autocorrelation times $\tau_{c} \leqslant \tau_{L}$, the sampling and interaction time of the field by the particle is reduced.

\section{Conclusion}

In this study, we revisited the radial diffusion formalism of planetary radiation belts by relaxing the assumption of zero correlation time typically found in stochastic acceleration models. We derived a diffusion coefficient and found selfsimilar solutions of the Fokker-Planck equation in the limit of short $\left(\tau_{L} \gg \tau_{c}\right)$ and long $\left(\tau_{L} \ll \tau_{c}\right)$ autocorrelation times. We showed that intermediate solutions of the Fokker-Planck equation for long autocorrelation time $\tau_{c}$ are subdiffusive with a variance that scales as $\left\langle\Delta \Phi^{2}\right\rangle \sim t^{1 / 4}$ and result in transport time independent of the drift frequency. On the other hand, intermediate solutions of the Fokker-Planck equation for short autocorrelation time $\tau_{c}$ depend on the drift frequency but result in slower subdiffusive transport with a variance that scales as $\left\langle\Delta \Phi^{2}\right\rangle \sim t^{1 / 2}$. However, at least in the absence of sources and sinks, particle transport for both short and long autocorrelation times result in stationary distribution along $L^{*}$ with differences of only less than $10 \%$ across lower magnetic drift shells. Thus, the presence of turbulent fluctuations with long autocorrelation time could result in faster diffusion of charged particles in planetary radiation belts during intermediate times and before the flattening of the distribution function across a broad range of magnetic drift shells.

Our results are also indicative of a diffusion coefficient that takes different parametric forms for short and long autocorrelation times. For $\tau_{c} \gg \Omega_{D}^{-1}$ the diffusion coefficient dependence on $L^{*}$ scales as $D_{L L} \simeq L^{* 10}$, whereas for $\tau_{c} \ll \Omega_{D}^{-1}$ the parametric dependence scales as $D_{L L} \simeq L^{* 10} \Omega_{D}^{2} \tau_{c}^{2} \sim L^{* 6} \tau_{c}^{2}$. Thus, the appearance of finite correlation times in the diffusion coefficient should result in a parametric dependence in the $L^{*}$ exponent that is less or equal than 10 . Such dependence should be apparent from observational studies inferring the parametric form of the diffusion coefficient. Using Bayesian inference and the Van Allen Probe measurements, Sarma et al. (2020) empirically determined the parametric dependence of the diffusion coefficient. They find a bimodal distribution for the exponent of $L^{*}$ in the diffusion coefficient, with one peak centered above a value of 10 and another, slightly more pronounced, below 10. Our study 
demonstrates that a spectrum of electromagnetic fluctuations with an admixture of short and long autocorrelation times would translate into a diffusion coefficient with a dependence in the $L^{*}$ exponent less and equal than 10 .

Despite the numerous simplifications (no drivers/sources or sinks, time homogeneity, exponential autocorrelation function, time-independent finite correlation time $\tau_{c}$, constant in time and space variance of the magnetic field fluctuations) our results demonstrate the rich range of dynamical behavior possible when only one of the assumptions for radial diffusion is relaxed. However, previous studies also indicate that additional assumptions, such as time homogeneity, are unjustified (Lejosne et al. 2013) and that the autocorrelation function of large-scale fluctuations is dependent on the geomagnetic activity. Thus, in future studies, we will incorporate a loss term and more realistic autocorrelation functions consistent with empirical observations and parameterized in terms of geomagnetic activity (Brautigam \& Albert 2000; Lejosne et al. 2013).

A.O. wishes to thank H. Koskinen, E. Kilpua, M. Kalliokoski, and $\mathrm{H}$. George for discussions on planetary radiation belts and A. A. Schekochihin and T. Adkins for discussions on stochastic acceleration in turbulent fields. A.O. acknowledges funding from the Academy of Finland by the profiling action on Matter and Materials, grant No. 318913. The work of S.L. was performed under JHU/APL Contract No. 922613 (RBSP-EFW) and NASA Grant Award 80NSSC18K1223.

\section{ORCID iDs}

Adnane Osmane (i) https://orcid.org/0000-0003-2555-5953

Solène Lejosne (iD https://orcid.org/0000-0003-4238-8579

\section{References}

Adkins, T. 2018, Masters Thesis, Rudolf Peierls Centre for Theoretical Physics Barenblatt, G. I. 1996, Scaling, Self-similarity, and Intermediate Asymptotics: Dimensional Analysis and Intermediate Asymptotics, Vol. 14 (Cambridge: Cambridge Univ. Press)

Bian, N. H., Kontar, E. P., \& Ratcliffe, H. 2014, JGRA, 119, 4239

Brautigam, D., \& Albert, J. 2000, JGRA, 105, 291

Diamond, P. H., Itoh, S.-I., \& Itoh, K. 2010, Modern Plasma Physics : Physical Kinetics of Turbulent Plasmas (Cambridge: Cambridge Univ. Press) Fälthammar, C.-G. 1965, JGR, 70, 2503

Huang, C. L., Spence, H. E., Hudson, M. K., \& Elkington, S. R. 2010, JGRA, 115, A06216

Lejosne, S. 2019, JGRA, 124, 4278

Lejosne, S., Boscher, D., Maget, V., \& Rolland, G. 2013, JGRA, 118, 3147

Lejosne, S., \& Kollmann, P. 2020, SSRv, 216, 1

Lichtenberg, A. J., \& Lieberman, M. A. 1983, Regular and Stochastic Motion (New York: Springer)

Mead, G. D. 1964, JGR, 69, 1181

Parker, E. 1960, JGR, 65, 3117

Reeves, G. D., Spence, H. E., Henderson, M. G., et al. 2013, Sci, 341, 991

Sarma, R., Chandorkar, M., Zhelavskaya, I., et al. 2020, JGRA, 125, e2019JA027618

Schulz, M., \& Eviatar, A. 1969, JGR, 74, 2182

Sturrock, P. A. 1966, PhRv, 141, 186

Taylor, G. I. 1922, Proc. London Math. Soc., s2-20, 196

Thorne, R. M. e. a. 2013, Natur, 504, 411

Ukhorskiy, A., \& Sitnov, M. 2013, SSRv, 179, 545

Walt, M. 2005, Introduction to Geomagnetically Trapped Radiation (Cambridge: Cambridge Univ. Press) 